\title{
The ever-expanding phenotypical spectrum of human TBX4 mutations: from toe to lung
}

\author{
Meindina G. Haarman ${ }^{1}$, Wilhelmina S. Kerstjens-Frederikse ${ }^{2}$ and \\ Rolf M.F. Berger ${ }^{1}$
}

Affiliations: ${ }^{1}$ Center for Congenital Heart Diseases, Dept of Pediatric Cardiology, Beatrix Children's Hospital, National Referral Center for Pediatric Pulmonary Hypertension, University Medical Center Groningen, University of Groningen, Groningen, The Netherlands. ${ }^{2}$ Dept of Genetics, University Medical Center Groningen, University of Groningen, Groningen, The Netherlands.

Correspondence: Rolf M.F. Berger, Beatrix Children's Hospital, University Medical Center Groningen, University of Groningen, Pediatric Cardiology, Hanzeplein 1, Groningen, The Netherlands.

E-mail: r.m.f.bergerQumcg.nl

@ERSpublications

Since the discovery of the TBX4 gene, the phenotypical spectrum of human TBX4 mutations is rapidly expanding from syndromes with skeletal dysplasia, to pulmonary hypertension to developmental lung diseases, all associated with disrupted organ development http://bit.ly/2MvYful

Cite this article as: Haarman MG, Kerstjens-Frederikse WS, Berger RMF. The ever-expanding phenotypical spectrum of human TBX4 mutations: from toe to lung. Eur Respir J 2019; 54: 1901504 [https://doi.org/10.1183/13993003.01504-2019].

Transcription factors of the T-box family are known to be involved in the regulation of embryonic developmental processes. T-box factor 4 (TBX4), one of its members first discovered in 1996, is expressed in a wide variety of tissues during organogenesis $[1,2]$. The TBX4 gene is located on chromosome 17, region q23.2 [3]. Most information on TBX4 defects have been obtained from animal models that have revealed that TBX4 plays a critical role, governing multiple processes during early limb and respiratory tract development. Loss of Tbx4 has been shown to block hindlimb and pelvic development, disrupts the development of the respiratory system and affects early embryonic vascularisation [4]. In mice, TBX4 (in concert with TBX5) regulates the process of lung branching by controlling the expression of the secreted fibroblast growth factor (FGF) 10 and activation of FGF10 signalling. Also, in the trachea, TBX4 and TBX5 are important for the formation of cartilage rings, although a distinct pathway that does not involve FGF10 regulates this [5]. The clinical phenotype of TBX4 defects in humans however, has begun only very recently to reveal itself (figure 1).

First, in 2002, mutations of the TBX4 gene were found to be associated with a spectrum of limb and skeletal abnormalities referred to as small patella syndrome (SPS), also known as ischiocoxopodopatellar syndrome. This autosomal-dominant skeletal dysplasia is characterised by aplasia or hypoplasia of the patella and developmental anomalies of the pelvis and feet [6]. The pathogenic mechanism by which TBX4 mutations cause SPS remains to be elucidated.

It was not before 2013 that TBX4 gene mutations were discovered to be associated with pulmonary arterial hypertension (PAH) in childhood [7]. In contrast to the identification of most other PAH-related genes, this discovery was primarily based on clinical observations, recognising phenotypical similarities in six children diagnosed with PAH considered idiopathic at diagnosis. In these children PAH was associated 
with unexplained dysmorphic features and mental retardation. Genetic analyses revealed overlapping 17 q23.2 deletions as a common denominator in three of them, where the minimal overlapping region of $1 \mathrm{Mb}$ contained several candidate genes including TBX4. Subsequent gene sequencing in an additional cohort of 14 paediatric patients diagnosed with idiopathic PAH but without associated dysmorphic features, identified TBX4 mutations in about $20 \%$ of these cases. Since then, the association of TBX4 mutations/deletion and PAH has been confirmed in different cohorts of paediatric PAH patients (idiopathic or hereditary) with a prevalence of approximately $7-10 \%$ [8, 9]. Although available data suggest a lower occurrence in adults with PAH (2-3\%), evidence is emerging that TBX4 gene mutations might form the second-most frequently mutated gene, after BMPRII, in both children and adults [10, 11].

Recently, incidental cases have been reported of neonates presenting with pulmonary hypertension and respiratory failure, due to various lung diseases, who were found to carry pathogenic TBX4 mutations. These included severe diffuse developmental lung disorders, such as acinar dysplasia and congenital alveolar dysplasia, conditions that are thought to represent a spectrum of growth arrest at different stages in lung development. The TBX4-FGF10-FGFR2 epithelial-mesenchymal signalling pathway has been recently suggested to play an important but complex role in neonatal disease conditions associated with lethal lung maldevelopment [12-14].

In the current issue of the European Respiratory Journal, GaLAmbos et al. [15] describe a selected series of 19 patients, collected from different institutions over the world, presenting with pulmonary hypertension and carrying different TBX4 variations, including mutations and deletions, and a variety of developmental lung disorders. With that the authors further define the ever-expanding spectrum of clinical manifestations and pulmonary histopathology associated with human TBX4 variations.

The authors report six children with 17q23 deletions and loss of TBX4 and 13 children with intragenic mutations. Interestingly, the majority of the children in this series (63\%) showed a biphasic clinical course presenting with persistent pulmonary hypertension of the newborn (PPHN) and respiratory failure with delusive resolution around the age of one month, followed by the emergence of chronic, progressive pulmonary hypertension later in infancy or early childhood. In seven patients, lung histopathology showed diffuse alveolar growth abnormalities and a variable degree of pulmonary arterial wall remodelling, with or without neointimal fibrosis. The severity of presentation of PPHN in early life seemed to correlate with more severe disruption of the distal lung development and contrasted with those who presented later in childhood.

Also in the current issue of the European Respiratory Journal, MAURAC et al. [16] report on a very interesting case of a 34-year-old woman, presenting with pulmonary hypertension, right heart failure and SPS, in who a TBX4 mutation was demonstrated. Although pulmonary function tests were unremarkable, except for a decreased carbon monoxide diffusion capacity, computed tomography imaging suggested bronchial and pulmonary parenchymal abnormalities. Lung biopsy tissue became available in this patient, showing pulmonary vascular remodelling corresponding with pulmonary hypertension, and in addition various airway and parenchymal abnormalities suggesting disturbed lung development.

A very interesting aspect of both these manuscripts was the availability of clinical course and lung histology in infants, children and adults with pulmonary hypertension associated with TBX4 mutations. All available tissue specimens showed, in addition to various degrees of pulmonary vascular hypertensive remodelling, histological features of abnormal distal lung development affecting alveolar, interstitial and vascular structures. These developmental lung abnormalities seem to form a spectrum with on one hand severe and diffuse features of growth arrest, including acinar dysplasia and congenital alveolar dysplasia, which are associated with severe and early neonatal clinical presentation, while on the other hand milder features of bronchial abnormalities, simplified alveolar development and interstitial remodelling that seem to be associated with presentation at post neonatal age, childhood or even adulthood $[15,16]$.

Accordingly, these reports show that the clinical course of TBX4 mutation-associated pulmonary hypertension is highly variable and seem to correspond with the degree of lung developmental disorder. Patients with TBX4 mutations and heritable PAH, thus without clinical signs of pulmonary bronchial or interstitial disorders, have been suggested to have a more favourable outcome compared to, for instance, those with BMPR2 mutations. However, genotype-phenotype relations within TBX4 mutations, as well as in comparison with other gene mutations, are still insufficiently studied.

The relationship between TBX4 gene mutations and developmental lung and bone disorders in humans is becoming increasingly clear and is not that surprising, given current knowledge derived predominantly from genetically manipulated animal models on the important roles of both TBX4 and TBX5 in the developing lung and trachea. However, the exact role of TBX4 in the development of the pulmonary structures, both the vasculature and the airways, is complex and currently insufficiently understood. This is 


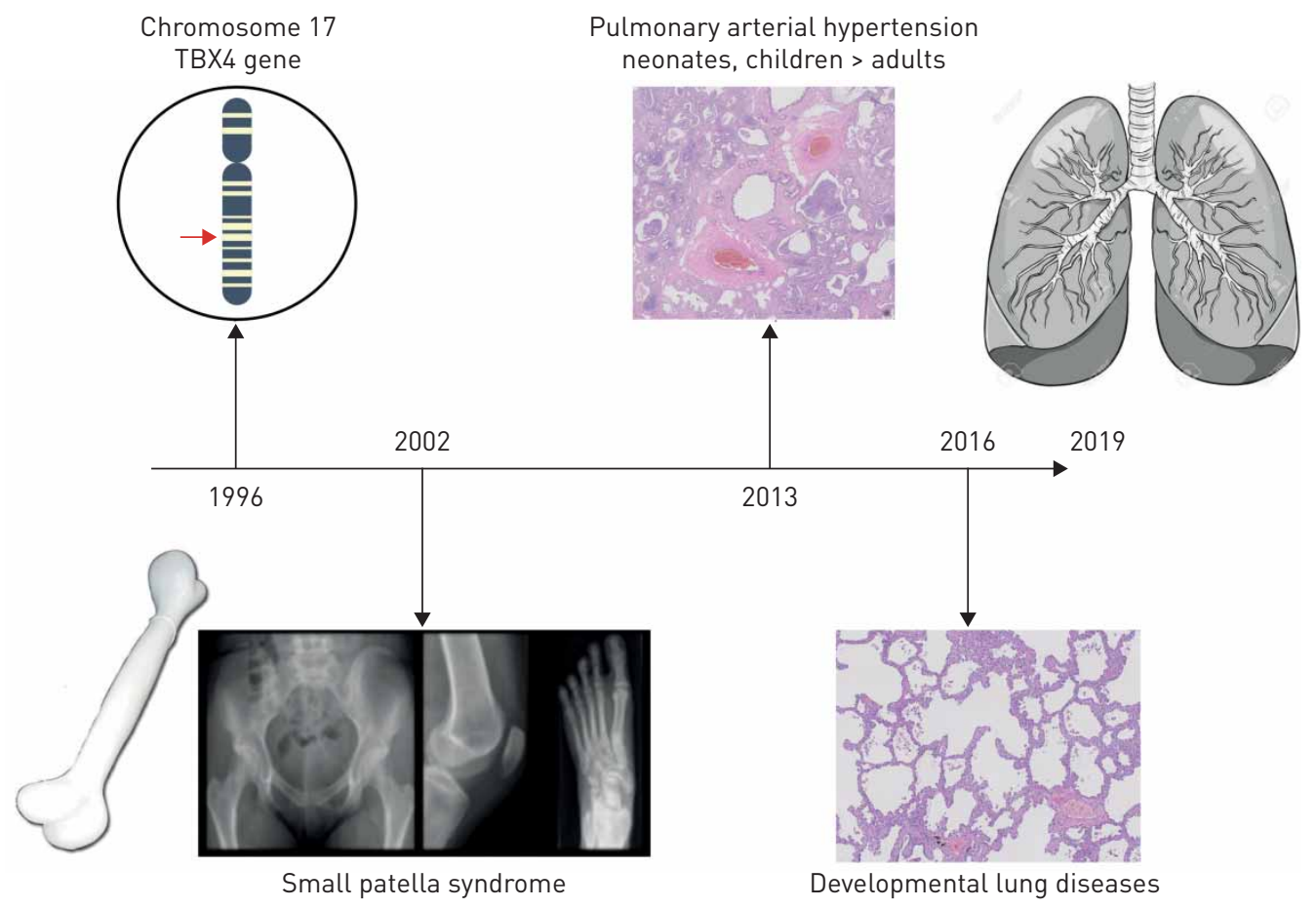

FIGURE 1 The ever-expanding phenotypical spectrum of TBX4 mutations since the discovery of the gene in 1996.

illustrated by the unexplained variety in penetrance between TBX4 mutation-associated bone anomalies (SPS), diseased pulmonary vasculature (PAH) or developmental lung disorders, as well the unexplained variety in expression between mutation carriers in the same family [6, 7]. TBX4 is known to affect the activity of members of the FGF-, Wnt- and BMP-pathways linking TBX4 mutations to the TGF- $\beta$ signalling pathway. Recent experimental observations indicate that TBX4 mutations may be associated with developmental disorders also in other organs than bones and lung, including anorectal malformations [17].

The emerging awareness that TBX4 variations are associated with both pulmonary hypertension and developmental parenchymal lung disease, potentially leading to respiratory compromise, challenges the diagnostic classification of pulmonary hypertension in a patient with TBX4 mutation. According to the clinical classification of pulmonary hypertension, proposed at the 2018 World Symposium of Pulmonary Hypertension, pulmonary hypertension associated with TBX4 mutations has been classified as group 1 $\mathrm{PAH}$, heritable PAH [18]. At the same time, the paediatric task force at this meeting suggested to include patients with pulmonary hypertension and a TBX4 mutation in group 3 pulmonary hypertension, associated with developmental lung disease [19]. Moreover, parenchymal lung disease may induce alveolar hypoxia inducing hypoxic vasoconstriction. In these particular patients, it seems even more obvious that a meticulous diagnostic work-up has to be performed in each individual patient in an attempt to distinguish $\mathrm{PAH}$ from pulmonary hypertension associated with hypoxia and/or disrupted development. In both cases, the presence of pulmonary hypertension will seriously affect clinical course and treatment most appropriate to the associated condition is mandatory.

In summary, Galambos et al. [15] and MaURaC et al. [16] must be commended for their contribution to the further comprehension of the still-emerging wide phenotypic spectrum associated with human TBX4 mutations. From neonate to adult, from bone to lung, mutations in the TBX4 gene are associated with developmental organ disorders, presenting with pulmonary hypertension that cannot be easily placed under one single denominator. Further studies on the mechanisms through which disturbed function of the TBX4 leads to pulmonary (arterial) hypertension and disrupted pulmonary development, are required in order to better understand phenotypic expression, inheritance and, ultimately, the optimal treatment approach of TBx4-associated pulmonary hypertension. 


\section{References}

1 Agulnik SI, Garvey N, Hancock S, et al. Evolution of mouse T-box genes by tandem duplication and cluster dispersion. Genetics 1996; 144: 249-254.

2 Chapman DL, Garvey N, Hancock S, et al. Expression of the T-box family genes, Tbx1-Tbx5, during early mouse development. Dev Dyn 1996; 206: 379-390.

3 Yi CH, Russ A, Brook JD. Virtual cloning and physical mapping of a human T-box gene, TBX4. Genomics 2000; 67: 92-95.

4 Naiche LA, Papaioannou VE. Loss of Tbx4 blocks hindlimb development and affects vascularization and fusion of the allantois. Development 2003; 130: 2681-2693.

5 Arora R, Metzger RJ, Papaioannou VE. Multiple roles and interactions of Tbx4 and Tbx5 in development of the respiratory system. PLoS Genet 2012; 8: e1002866.

6 Bongers EMHF, Duijf PHG, van Beersum SEM, et al. Mutations in the human TBX4 gene cause small patella syndrome. Am J Hum Genet 2004; 74: 1239-1248.

7 Kerstjens-Frederikse WS, Bongers EMHF, Roofthooft MTR, et al. TBX4 mutations (small patella syndrome) are associated with childhood-onset pulmonary arterial hypertension. J Med Genet 2013; 50: 500-506.

8 Levy M, Eyries M, Szezepanski I, et al. Genetic analyses in a cohort of children with pulmonary hypertension. Eur Respir J 2016; 48: 1118-1126.

9 Zhu N, Gonzaga-Jauregui C, Welch CL, et al. Exome sequencing in children with pulmonary arterial hypertension demonstrates differences compared with adults. Circ Genomic Precis Med 2018; 11: e001887.

10 Navas P, Tenorio J, Quezada CA, et al. Molecular analysis of BMPR2, TBX4, and KCNK3 and genotype-phenotype correlations in Spanish patients and families with idiopathic and hereditary pulmonary arterial hypertension. Rev Esp Cardiol (Engl Ed) 2016; 69: 1011-1019.

11 Eyries M, Montani D, Nadaud S, et al. Widening the landscape of heritable pulmonary hypertension mutations in paediatric and adult cases. Eur Respir J 2018; 53: 1801371.

12 Suhrie K, Pajor NM, Ahlfeld SK, et al. Neonatal lung disease associated with TBX4 mutations. J Pediatr 2019; 206 : 286-292. e1.

13 Szafranski P, Coban-Akdemir ZH, Rupps R, et al. Phenotypic expansion of TBX4 mutations to include acinar dysplasia of the lungs. Am J Med Genet A 2016; 170: 2440-2444.

14 Karolak JA, Vincent M, Stankiewicz P, et al. Complex compound inheritance of lethal lung developmental disorders due to disruption of the TBX-FGF pathway. Am J Hum Genet 2019; 104: 213-228.

15 Galambos C, Mullen MP, Shieh JT, et al. Phenotype characterisation of TBX4 mutation and deletion carriers with neonatal and paediatric pulmonary hypertension. Eur Respir J 2019; 54: 1801965.

16 Maurac A, Lardenois É, Eyries M, et al. T-box protein 4 mutation causing pulmonary arterial hypertension and lung disease. Eur Respir J 2019; 54: 1900388.

17 Li M, Zhang $\mathrm{H}$, Liu $\mathrm{H}$, et al. Abnormal expression of TBX4 during anorectal development in rat embryos with ethylenethiourea-induced anorectal malformations. Biol Res 2019; 52: 27.

18 Simonneau G, Montani D, Celermajer DS, et al. Haemodynamic definitions and updated clinical classification of pulmonary hypertension. Eur Respir J 2019; 53: 1801913.

19 Rosenzweig EB, Abman SH, Adatia I, et al. Paediatric pulmonary arterial hypertension: updates on definition, classification, diagnostics and management. Eur Respir J 2019; 53: 1801916. 